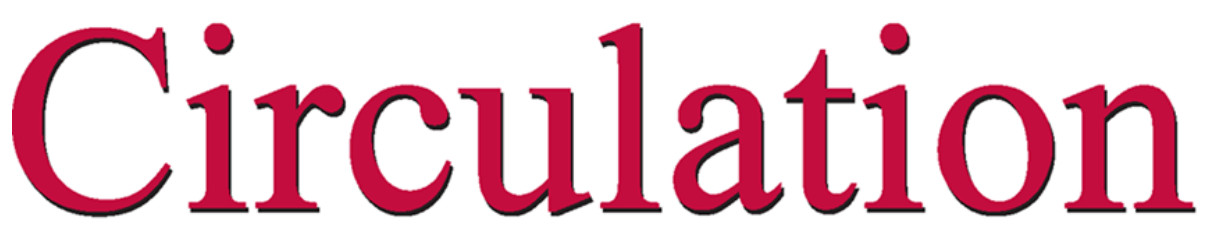

JOURNAL OF THE AMERICAN HEART ASSOCIATION
American Heart Association

Learn and Live

\title{
Sirolimus-Eluting Stent for the Treatment of In-Stent Restenosis: A Quantitative Coronary Angiography and Three-Dimensional Intravascular Ultrasound Study
}

J. Eduardo Sousa, Marco A. Costa, Alexandre Abizaid, Amanda G.M.R. Sousa, Fausto Feres, Luiz A. Mattos, Marinella Centemero, Galo Maldonado, Andrea S. Abizaid, Ibraim Pinto, Robert Falotico, Judith Jaeger, Jeffrey J. Popma and Patrick W. Serruys

Circulation 2003;107;24-27; originally published online Dec 2, 2002; DOI: 10.1161/01.CIR.0000047063.22006.41

Circulation is published by the American Heart Association. 7272 Greenville Avenue, Dallas, TX 72514

Copyright @ 2003 American Heart Association. All rights reserved. Print ISSN: 0009-7322. Online ISSN: $1524-4539$

The online version of this article, along with updated information and services, is located on the World Wide Web at: http://circ.ahajournals.org/cgi/content/full/107/1/24

Subscriptions: Information about subscribing to Circulation is online at http://circ.ahajournals.org/subsriptions/

Permissions: Permissions \& Rights Desk, Lippincott Williams \& Wilkins, 351 West Camden Street, Baltimore, MD 21202-2436. Phone 410-5280-4050. Fax: 410-528-8550. Email: journalpermissions@1ww.com

Reprints: Information about reprints can be found online at http://www.lww.com/static/html/reprints.html 


\title{
Sirolimus-Eluting Stent for the Treatment of In-Stent Restenosis A Quantitative Coronary Angiography and Three-Dimensional Intravascular Ultrasound Study
}

\author{
J. Eduardo Sousa, MD, PhD; Marco A. Costa, MD, PhD; Alexandre Abizaid, MD, PhD;
} Amanda G.M.R. Sousa, MD, PhD; Fausto Feres, MD, PhD; Luiz A. Mattos, MD, PhD; Marinella Centemero, MD; Galo Maldonado, MD; Andrea S. Abizaid, MD; Ibraim Pinto, MD; Robert Falotico, PhD; Judith Jaeger, BA; Jeffrey J. Popma, MD; Patrick W. Serruys, MD, PhD

Background - We have previously reported the safety and effectiveness of sirolimus-eluting stents for the treatment of de novo coronary lesions. The present investigation explored the potential of this technology to treat in-stent restenosis. Methods and Results - Twenty-five patients with in-stent restenosis were successfully treated with the implantation of 1 or 2 sirolimus-eluting Bx VELOCITY stents in São Paulo, Brazil. Nine patients received 2 stents (1.4 stents per lesion). Angiographic and volumetric intravascular ultrasound (IVUS) images were obtained after the procedure and at 4 and 12 months. All vessels were patent at the time of 12-month angiography. Angiographic late loss averaged $0.07 \pm 0.2 \mathrm{~mm}$ in-stent and $-0.05 \pm 0.3 \mathrm{~mm}$ in-lesion at 4 months, and $0.36 \pm 0.46 \mathrm{~mm}$ in-stent and $0.16 \pm 0.42 \mathrm{~mm}$ in-lesion after 12 months. No patient had in-stent or stent margin restenosis at 4 months, and only one patient developed in-stent restenosis at 1-year follow-up. Intimal hyperplasia by 3-dimensional IVUS was $0.92 \pm 1.9 \mathrm{~mm}^{3}$ at 4 months and $2.55 \pm 4.9 \mathrm{~mm}^{3}$ after 1 year. Percent volume obstruction was $0.81 \pm 1.7 \%$ and $1.76 \pm 3.4 \%$ at the 4 - and 12-month follow-up, respectively. There was no evidence of stent malapposition either acutely or in the follow-up IVUS images, and there were no deaths, stent thromboses, or repeat revascularizations.

Conclusion - This study demonstrates the safety and the potential utility of sirolimus-eluting Bx VELOCITY stents for the treatment of in-stent restenosis. (Circulation. 2003;107:24-27.)

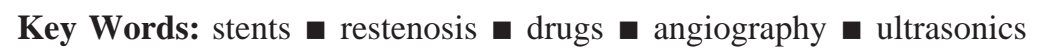

$\mathrm{T}$ reating in-stent restenosis (ISR) has become one of the major challenges for the interventional cardiologist. ${ }^{1}$ Regardless of which percutaneous approach is chosen to treat an in-stent restenotic lesion (balloon angioplasty, stent, rotational atherectomy, or laser angioplasty), $30 \%$ to $80 \%$ of the patients will develop restenosis within the stent, at the stent edges, or both. Currently, the only proven effective therapy available for patients with ISR is intravascular brachytherapy. ${ }^{2}$ Unfortunately, this complex therapy carries with it the risks associated with ionizing radiation, including delayed endothelialization and potential late vascular thrombosis. ${ }^{3}$

The first-in-man (FIM) study has recently demonstrated the safety and effectiveness of sirolimus-eluting Bx VELOCITY stents for the treatment of single, de novo coronary lesions. ${ }^{4,5}$ In this somewhat benign population, no major clinical events (thrombosis, repeat revascularization, myocardial infarction, or death) occurred within 1-year of follow-up. Twelve months after the index procedure, neointimal prolif- eration and late lumen loss were virtually absent as determined both by quantitative intravascular ultrasound (IVUS) and angiography. Subsequently, the multicenter RAndomized study with the sirolimus-eluting $\mathrm{Bx}$ VELocity balloonexpandable stent (RAVEL) study corroborated these results by reporting the absence of restenosis 6 months after the implantation of sirolimus-eluting stents in a similar subset of patients with de novo, single vessel, coronary artery disease. ${ }^{6}$ Whether such unparalleled results would be replicated in a more complex patient group, such as those with ISR, remains to be determined.

The aims of this pilot study were to determine the feasibility and safety of treating ISR with slow-release sirolimuseluting Bx VELOCITY stents and to determine the impact of this technology on prevention of recurrent restenosis.

\section{Methods}

From March to June of 2001, 25 consecutive patients with ISR were successfully treated with the implantation of the sirolimus-eluting Bx

Received August 28, 2002; revision received October 28, 2002; accepted October 30, 2002.

From the Institute Dante Pazzanese of Cardiology, São Paulo, Brazil (J.E.S., A.A., A.G.M.R.S., F.F., L.A.M., M.C., G.M., A.S.A., I.P.); University of Florida-Shands, Jacksonville (M.A.C.); Cordis, a Johnson \& Johnson Company, Warren, NJ (R.F., J.J.); Brigham and Women's Hospital, Boston, Mass (J.J.P.); and Thoraxcenter, Dijkzigt University Hospital, Rotterdam, The Netherlands (P.W.S.).

Correspondence to Prof J. Eduardo Sousa, MD, PhD, Director of the Institute Dante Pazzanese of Cardiology, Av. Dr Dante Pazzanese, 500 Ibirapuera, 04012180, São Paulo, Brazil. E-mail jesousa@uol.com.br

(C) 2003 American Heart Association, Inc. 
TABLE 1. Quantitative Coronary Angiographic Analysis

\begin{tabular}{|c|c|c|c|c|}
\hline Parameters & Preprocedure & Postprocedure & 4-Month Follow-Up & 12-Month Follow-Up \\
\hline $\mathrm{RD}, \mathrm{mm}$ & $2.78 \pm 0.3$ & $2.8 \pm 0.4$ & $2.84 \pm 0.3$ & $2.8 \pm 0.3$ \\
\hline MLD, mm & $1.05 \pm 0.3$ & $\ldots$ & $\ldots$ & $\ldots$ \\
\hline DS, $\%$ & $62.2 \pm 10.5$ & $\ldots$ & $\ldots$ & $\ldots$ \\
\hline Lesion length, mm & $13.6 \pm 7.65$ & $\ldots$ & $\ldots$ & $\cdots$ \\
\hline In-stent MLD, mm & $\ldots$ & $2.71 \pm 0.3$ & $2.64 \pm 0.4$ & $2.35 \pm 0.6^{\star}$ \\
\hline In-lesion MLD, mm & $\ldots$ & $2.34 \pm 0.4$ & $2.39 \pm 0.4$ & $2.18 \pm 0.6 \dagger$ \\
\hline In-stent DS, \% & $\ldots$ & $2.6 \pm 9$ & $7.15 \pm 8.57$ & $16.7 \pm 17.7$ \\
\hline In-lesion DS, \% & $\ldots$ & $16.5 \pm 8.2$ & $15.96 \pm 9.1$ & $22.9 \pm 16.9$ \\
\hline In-stent late loss, mm & $\ldots$ & $\ldots$ & $0.07 \pm 0.2$ & $0.36 \pm 0.46$ \\
\hline In-lesion late loss, mm & $\ldots$ & $\ldots$ & $-0.05 \pm 0.3$ & $0.16 \pm 0.42$ \\
\hline
\end{tabular}

VELOCITY stent. Stents were coated with $140 \mu \mathrm{g}$ sirolimus $/ \mathrm{cm}^{2}$ per unit of metal surface area. The slow release formulation ( $\geq 28$-day drug release) was used in this protocol. ${ }^{4,5}$ Patients were excluded if they had undergone previous intravascular radiation therapy to the target vessel, if the index lesion was $>36 \mathrm{~mm}$ in length, or if the lesion was located in a saphenous vein graft.

\section{Procedure}

All stents were $18 \mathrm{~mm}$ long and varied from 2.5 to $3.5 \mathrm{~mm}$ in diameter. There was no ostial lesion treated in the present investigation. After predilatation of the target lesion, stents were deployed with high-pressure ( $>14$ atmospheres) postdilatation, guided by IVUS to assure complete stent expansion, which was defined as in-stent minimal lumen area $>80 \%$ of reference lumen area, and apposition. Debulking devices were not used, and predilatation was performed with conventional balloon. The operator was allowed to implant up to 2 sirolimus-eluting stents to cover the entire length of the lesion, but coverage of the entire stented segment was not mandatory. Overlapping of the 2 adjacent sirolimus-eluting stents was recommended per the protocol. All patients received aspirin ( $325 \mathrm{mg} / \mathrm{d}$, indefinitely) commencing at least 12 hours before the procedure and clopidogrel $300 \mathrm{mg}$ immediately after stent implantation and $75 \mathrm{mg} / \mathrm{d}$ for 60 days thereafter. The protocol was approved by the Medical Ethical Committee and informed consent was obtained for every patient.

\section{Quantitative Measurements}

Postprocedure and 4- and 12-month quantitative coronary angiography (QCA) and IVUS imaging were performed in all patients. IVUS images were acquired using a motorized pullback at a constant speed of $0.5 \mathrm{~mm} / \mathrm{sec}$ and volumetric quantification was performed. Quantitative angiographic and volumetric IVUS analyses were performed by independent Core laboratories (Brigham and Women's Hospital, Boston, Mass and Cardialysis B.V, Rotterdam, The Netherlands, respectively). The three segments selected for volumetric IVUS analysis were the segment covered by sirolimus-eluting stents and 2 edge segments (axially $5 \mathrm{~mm}$ proximal and distal to the sirolimuseluting stent margins). The presence of intimal hyperplasia in previously deployed stents and anatomical landmarks noted by angiography during stent deployment were used to guide IVUS selection of the segment covered by sirolimus-eluting stents.

As described previously, 52 coronary segments were subjected to quantitative angiography, in-stent and in-lesion segments. The instent analysis encompassed only the segment covered by sirolimuseluting stent. The in-lesion segment was defined as the in-stent segment plus segments 5-mm proximal and 5-mm distal to the edge or the nearest side branch if the side branch was nearer than $5 \mathrm{~mm}$ from the stent edge. Contrast injection and angiography was performed after positioning the stent delivery system just before deployment. The relation between anatomic landmarks, including the previously deployed stents, and the 2 radiopaque markers of the delivery system were noted. In-stent and in-lesion restenosis were defined as $\geq 50 \%$ diameter stenosis (DS) at follow-up, located within the stent and target lesion, respectively. Minimal lumen diameter (MLD) and \%DS were measured for each segment. In-lesion and in-stent late lumen loss (LL) were calculated as postprocedure MLD minus follow-up MLD. Validation of volumetric IVUS quantification has been described elsewhere. ${ }^{7}$

\section{Statistical Analysis}

Continuous variables are expressed as mean \pm SD. Comparisons between postintervention and follow-up measurements were performed with a 2 -tailed paired $t$ test. A probability value $<0.05$ was considered statistically significant.

\section{Results}

The mean age was $56 \pm 12$ years; $80 \%$ of the patients were male, and $24 \%$ were diabetics. Five patients $(20 \%)$ had recurrent ISR. The time interval between initial bare metal stent implantation and development of in-stent restenosis varied from 3 to 7 months. Lesions were classified as focal $(\leq 10 \mathrm{~mm})$ in $32 \%$, diffuse intrastent in $40 \%$, and diffuse proliferative in $28 \%$ of the patients according to previously reported classification. ${ }^{1}$ All stents were implanted successfully. Stent edge dissection was not detected either by angiography or IVUS and patients were discharged without complications 24 hours after treatment.

All patients were free of angina after 1 year, and there were no repeat revascularizations, stent thromboses, or major adverse clinical events (cerebrovascular accident, myocardial infarction, or death) during this time.

Angiographic data are presented in Table 1. At 4 months, in-stent lumen diameter remained essentially unchanged from postprocedure in $50 \%$ of the cases (Figure 1). Minimal angiography lumen gain observed in some patients may be explained by regression of intimal hyperplasia that was not covered with the sirolimus-eluting stent. There was a slightly but statistically significant decrease in in-stent MLD between 4 and 12 months (Table 1, Figure 1), whereas in-lesion MLD was essentially unchanged after 12 months (Table 1, Figure 1). Only 1 patient, who was asymptomatic, developed in-stent reste- 


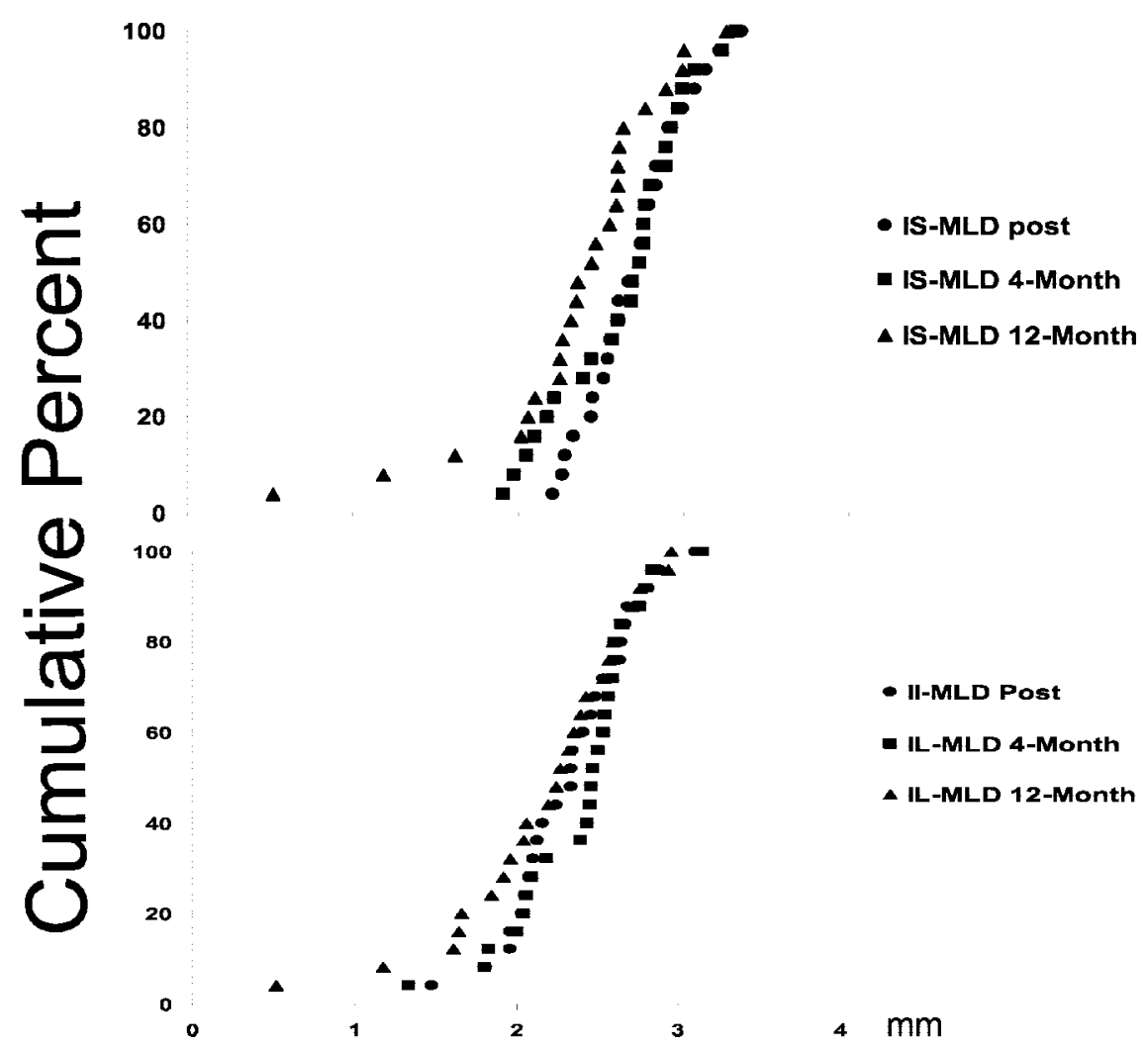

Figure 1. Cumulative distribution curves of angiographic in-stent (IS, upper panel) and in-lesion (IL, lower panel) minimal lumen diameter (MLD) after the procedure (Post) and at 4-month and 12-month follow-ups.

nosis at 12 months. IVUS imaging was not performed in this patient. IVUS analysis showed minimal neointimal hyperplasia volume $\left(0.92 \pm 1.9 \mathrm{~mm}^{3}\right.$ at 4 months and $2.55 \pm 4.9 \mathrm{~mm}^{3}$ at 12 months) in the remaining patients (Table 2).

\section{Discussion}

The present study demonstrates the feasibility of using sirolimus-eluting stents for the treatment of ISR. All patients were asymptomatic, and only 1 patient developed in-stent restenosis 12 months after treatment. In-stent intimal hyperplasia was minimal in most patients (Figure 2), and the in-lesion late loss was only $0.16-\mathrm{mm}$. Most importantly, the 12-month clinical follow-up was uneventful for all patients.

A number of clinical studies have documented the challenge of treating ISR because of the high recurrence of restenosis $(30 \%$ to $80 \%),{ }^{1,8}$ which is directly related to the length of the ISR lesion and independent of device selection. Only recently has catheter-based intravascular brachytherapy become available as an effective treatment for patients with ISR. ${ }^{2}$ Although intravascular brachytherapy trials have consistently shown a $40 \%$ to $70 \%$ reduction in re-restenosis as compared with placebo, there is still a $17 \%$ to $32 \%$ long-term failure rate for patients treated with brachytherapy. $2,9,10$ The pioneer Scripps Coronary Radiation to Inhibit Proliferation Post Stenting (SCRIPPS) study irradiated 26 patients $(62 \%$ had ISR) using the Iridium-192 gamma-source. ${ }^{2}$ Lesion length was $<10 \mathrm{~mm}$ in $42 \%$ of the patients. Out of the 24 patients with complete angiographic follow-up, $17 \%$ had recurrent restenosis.

In the present study, in which $68 \%$ of the patients had diffuse ISR, there was no in-stent or edge restenosis 4 months after the implantation of sirolimus-eluting stents, and only 1 patient developed restenosis at 12 months. This patient had a

TABLE 2. Quantitative Intravascular Ultrasound Analysis

\begin{tabular}{lccc}
\hline Parameters & Postprocedure & 4-Month Follow-Up & 12-Month Follow-Up* \\
\hline Stented segment length, $\mathrm{mm}$ & $21.6 \pm 7.4$ & $21.6 \pm 7.5$ & $21.9 \pm 7.34$ \\
Mean lumen area, $\mathrm{mm}^{2}$ & $6.74 \pm 1.82$ & $6.81 \pm 1.9$ & $7.06 \pm 1.81$ \\
Percent obstruction volume, $\%$ & $\ldots$ & $0.81 \pm 1.7$ & $1.76 \pm 3.44$ \\
Peri-stent margins & & & \\
$\quad$ Distal mean lumen area, $\mathrm{mm}^{2}$ & $7.04 \pm 2.5$ & $7.27 \pm 2.7$ & $7.24 \pm 2.43$ \\
$\quad$ Proximal mean lumen area, $\mathrm{mm}^{2}$ & $7.46 \pm 2.39$ & $8 \pm 2.78$ & $8 \pm 2.59$ \\
$\quad$ Distal mean total vessel area, $\mathrm{mm}^{2}$ & $12.64 \pm 5.16$ & $12.69 \pm 4.94$ & $12.99 \pm 5.15$ \\
Proximal mean total vessel area, $\mathrm{mm}^{2}$ & $15.35 \pm 3.85$ & $15.99 \pm 4.11$ & $15.54 \pm 4.29$ \\
\hline
\end{tabular}

*Data do not include 1 patient with in-stent restenosis in whom the lesion could not be crossed with the IVUS catheter. 


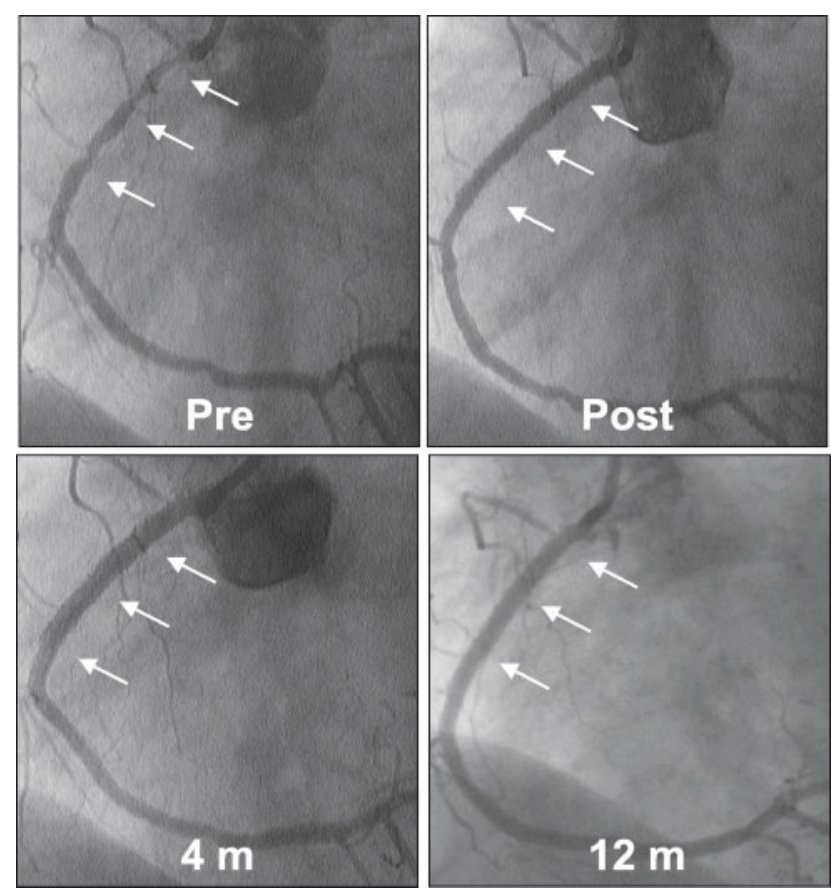

Figure 2. Preprocedure, postprocedure, 4-month, and 12-month follow-up angiography of a right coronary diffuse ISR lesion treated with 2 sirolimus-eluting stents. Lumen dimensions remained unchanged at 4-month and 12-month follow-up (bottom).

saphenous vein graft to the right coronary artery, and the $80 \%$ DS repeat ISR was located in the native artery distally to the site of graft anastomosis. Whether the proximity of the vein graft influenced the angiographic outcome remains to be clarified. The average change in MLD between 4 and 12 months shown by QCA was largely driven by this and one other patient who showed additional late loss (Figure 1), rather than by a systematic increase in late loss in all 25 patients.

Although clopidogrel was given for only 60 days after the procedure, there were no silent or overt stent thromboses as assessed by angiography and IVUS at 4 and 12 months, which was a 10-month period off dual antiplatelet therapy. This is also consistent with the observations in the RAVEL study, where patients have been off dual antiplatelet agents for at least 10 months without thrombotic events. ${ }^{6}$ This should be compared with the $\geq 6 \%$ late thrombosis rate observed after intracoronary radiation therapy, ${ }^{3}$ which resulted in the recommendation for prolonged antiplatelet therapy (6 to 12 months) after brachytherapy, especially when a new stent is implanted at the time of the procedure. The outcomes of patients with ISR treated with stents covered with a polymer containing a paclitaxel derivative have been reported recently. ${ }^{11}$ Although the results seemed promising after 6 months, 8 out of 13 patients had restenosis at 12 months and 2 patients experienced non-Q-wave myocardial infarctions due to stent occlusion. ${ }^{11}$
The lack of a control group clearly precludes a definitive scientific assessment of the excellent outcomes observed in the present study. The lack of stent thrombosis, repeat revascularization, myocardial infarction, or death in this patient population, however, indicates that the antiproliferative and antiinflammatory actions of sirolimus ${ }^{12}$ may provide a long-lasting antirestenosis effects after treatment of ISR lesions without the attendant risk of stent thrombosis observed with other effective treatment modalities. Longer-term follow-up will be required to determine whether the inhibition of in-stent restenosis observed in this study is sustained.

Although these data in a small cohort of patients are very encouraging, randomized controlled trials will be necessary to determine whether the reduction in late lumen loss, restenosis, and need for repeat intervention is indeed better than with brachytherapy, is sustained over several years, and can be achieved without the need for extended antiplatelet therapy.

\section{Acknowledgments}

We thank Drs Brian Firth and Dennis Donohoe for their careful review of the manuscript.

\section{References}

1. Mehran R, Dangas G, Abizaid AS, et al. Angiographic patterns of in-stent restenosis: classification and implications for long-term outcome. Circulation. 1999;100:1872-1878.

2. Teirstein PS, Massullo V, Jani S, et al. Catheter-based radiotherapy to inhibit restenosis after coronary stenting. $N$ Engl J Med. 1997;336: 1697-1703.

3. Costa MA, Sabate M, van der Giessen WJ, et al. Late coronary occlusion after intracoronary brachytherapy. Circulation. 1999;100:789-792.

4. Sousa JE, Costa MA, Abizaid A, et al. Lack of neointimal proliferation after implantation of sirolimus-coated stents in human coronary arteries: a quantitative coronary angiography and three-dimensional intravascular ultrasound study. Circulation. 2001;103:192-195.

5. Sousa JE, Costa MA, Abizaid AC, et al. Sustained suppression of neointimal proliferation by sirolimus-eluting stents: one-year angiographic and intravascular ultrasound follow-up. Circulation. 2001;104: 2007-2011.

6. Morice MC, Serruys PW, Sousa JE, et al. A randomized comparison of a sirolimus-eluting stent with a standard stent for coronary revascularization. N Engl J Med. 2002;346:1773-1780.

7. von Birgelen C, de Vrey EA, Mintz GS, et al. ECG-gated threedimensional intravascular ultrasound: feasibility and reproducibility of the automated analysis of coronary lumen and atherosclerotic plaque dimensions in humans. Circulation. 1997;96:2944-2952.

8. Lowe HC, Oesterle SN, Khachigian LM. Coronary in-stent restenosis: current status and future strategies. J Am Coll Cardiol. 2002;39:183-193.

9. Waksman R, White RL, Chan RC, et al. Intracoronary gamma-radiation therapy after angioplasty inhibits recurrence in patients with in-stent restenosis. Circulation. 2000;101:2165-2171.

10. Leon MB, Teirstein PS, Moses JW, et al. Localized intracoronary gamma-radiation therapy to inhibit the recurrence of restenosis after stenting. N Engl J Med. 2001;344:250-256.

11. Liistro F, Stankovic G, Di Mario C, et al. First clinical experience with a paclitaxel derivate-eluting polymer stent system implantation for in-stent restenosis: immediate and long- term clinical and angiographic outcome. Circulation. 2002;105:1883-1886.

12. Suzuki T, Kopia G, Hayashi S-i, et al. Stent-based delivery of sirolimus reduces neointimal formation in a porcine coronary model. Circulation. 2001;104:1188-1193. 